\title{
L'ECHANGE DE PAROLES SUR LA SEXUALITE : UNE ANALYSE DES RELATIONS DE CONFIDENCE
}

\author{
Alexis FERRAND, Lise MOUNIER \\ In Population, 1993, n 5, 1451-1476
}

\section{RESUME}

Pour analyser l'influence de l'environnement relationnel sur la façon dont les individus établissent leurs relations sexuelles, nous avons demandé aux enquêtés de décrire trois de leurs confidents en matière amoureuse et sexuelle. L'article présente les dimensions principales des logiques sociales de la confidence à partir de trois points de vue : celui de la prédisposition d'individus de différentes catégories à avoir plus ou moins de confidents de tels ou tels types ; celui des matrices d'échanges entre catégories d'acteurs résultant des relations ; celui des différents types de lien autorisant des échanges de confidence.

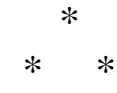

Dans son "Rapport sur le Sida", remis au terme d'une mission marathon en Octobre 1988, le Professeur GOT notait l'insuffisance des connaissances sur les dimensions sociales et psychologiques de la sexualité humaine. Et il faut reconnaître que rares étaient les chercheurs qui avaient étudié les comportements sexuels en tant que tels ${ }^{1}$. Ce sont donc souvent des connaissances et des problématiques jusqu'alors appliquées à d'autres objets qui ont été mobilisées pour tenter de mieux comprendre les comportements sexuels.

\subsection{La sexualité : comportements ou relations ?}

La démarche que nous avons adoptée résulte d'un transfert de ce type. Elle provient de recherches sur les relations d'amitié et la sociabilité menées depuis le milieu des années 1980 et utilisant une méthode d'analyse des réseaux personnels (FERRAND, 1985, 1987). De l'amitié à l'amour, le fossé ne semblait pas infranchissable.

L'idée centrale est simple. La sociologie des "rôles" met surtout l'accent sur les comportements institutionnalisés, sur des interactions gouvernées par les normes propres à un cadre social particulier, normes que l'individu aurait intériorisées. Que dire d'une relation interpersonnelle romantiquement amoureuse ou crûment sexuelle ? Existe-t-il un "rôle" de l'amant ? Certes, la conscience sociale abonde en images fortes. Mais ce sont des images variées,

\footnotetext{
${ }^{1}$ Ce sont les inscriptions légitimes et institutionnalisées de la sexualité (famille, parenté) qui polarisaient l'attention.
} 
des rôles contrastés, plutôt qu'un rôle. Les relations interindividuelles réelles suivent-elles ces modèles ? De fort loin. Les acteurs eux-mêmes peuvent jouer sur un écart entre la définition plus ou moins idéale qu'ils adoptent et leur propre pratique (BIDART, 1991).

La sociologie interactionniste met en question l'évidence a priori des rôles pour insister sur la dynamique complexe d'ajustement réciproque des acteurs (ROSE, 1962). De plus une relation comporte une diversité et une succession d'interactions : c'est un processus évolutif entre des acteurs et non une réalité figée ou répétitive (LAZARSFELD, MERTON, 1954). Processus au cours duquel les protagonistes négocient, de manière implicite ou explicite, ce qui est "convenable" pour leur relation au fur et à mesure que celle-ci évolue (ALLAN, 1979). D'où il résulte une grande variété des contenus relationnels, aisément repérables si les protocoles d'enquête offrent aux sujets une diversité de réponses suffisante (FERRAND, 1989b).

Dans cette vision interactionniste, on renonce à envisager le "comportement sexuel" d'un acteur. C'est la relation, la rencontre de deux acteurs, les accords et désaccords auxquels ils parviennent qui constituent l'objet à analyser. D'ailleurs, le simple bon sens enseigne que, sans en épuiser la signification totale, la vie sexuelle est faite de "rapports" et de "relations" : il y a le plus souvent deux protagonistes qui, bon gré mal gré, "font des choses ensemble".

\subsubsection{LE ROLE DES NORMES DANS LA NEGOCIATION ENTRE PARTENAIRES}

Pour comprendre la dynamique de cet "ensemble" formé par les deux partenaires, différents points de vue peuvent être adoptés (PETO et alii, 1992²). Par exemple, on peut examiner le rôle joué par la diversité et la confrontation des attentes des protagonistes ; ou par les écarts entre les ressources sociales dont ils disposent et les investissements faits dans la relation, qui induisent la capacité d'un protagoniste à faire prévaloir ses demandes ; ou encore par les normes régulant la relation elle-même ("équité" / "égalité").

En réponse aux sollicitations de recherche sur les comportements sexuels 3 nous avons retenu les "normes" et les interactions comme une dimension particulière et un enjeu des négociations entre protagonistes. Entre l'urgence incontrôlable du désir et le froid calcul du risque par un acteur informé et rationnel, une des voies par lesquelles se manifeste le caractère socialisé des acteurs n'est-elle pas l'existence de "normes" ? Entre le désirable et l'évaluable, il y aurait place pour le recommandable et le condamnable.

Supposer un effet des "normes" sociales, n'est-ce pas revenir à une perspective fonctionnaliste ? Tout dépend de la conception que l'on adopte des normes et de leurs modes d'intervention dans la conduite des acteurs. Quelques postulats ont orienté notre recherche.

\footnotetext{
2 Les "facteurs d'intelligibilité de la typologie", dans la présentation de cette recherche, mettent en jeu simultanément des propriétés individuelles, interindividuelles (dyadiques) et des réseaux relationnels ; le passage de la "simultanéité" à l'intégration théorique demeure un chantier ouvert pour des travaux ultérieurs.

3 Ces sollicitations sont apparues fin 1988 au sein du CNRS (secteur SHS), puis ont été formalisées par le premier appel d'offre de l'Agence Nationale de Recherches sur le Sida en avril 1989. Voir l'article de N. Bajos et A. Spira, Population, $1993, n^{\circ} 5$.
} 
- Les "normes" ne sont pas des préceptes stables, plus ou moins bien intériorisés au cours d'une socialisation juvénile, mais des principes de conduite qui subissent des réajustements au cours de la vie.

- Ces principes interviennent dans les relations interindividuelles pour orienter la manière dont un acteur est "reconnu" par des pairs ; et tout acteur est plus sensible à ces appréciations et jugements "proches" qu'à un écart entre son comportement et des normes générales et abstraites.

- Les acteurs peuvent vivre dans des environnements relationnels (cercles sociaux) et entretenir des relations qui comportent des principes de conduite contrastés, voire contradictoires.

- Des contextes relationnels contradictoires peuvent mettre l'acteur en position instable, favoriser des changements (pouvant aller aussi bien dans un "bon" qu'un "mauvais" sens, du point de vue de la prévention) ${ }^{4}$.

\subsubsection{DES PROCHES INFLUENTS : LES CONFIDENTS}

Pour analyser ces "influences de l'environnement relationnel" deux questions majeures doivent être tranchées : quelles relations influencent significativement la vie sexuelle ? Comment s'exerce cette influence?

La façon dont un acteur est sensible au jugement d'autrui peut être envisagée de manière diverse. D'un côté on imaginera que ce sont les opinions explicites, les principes déclarés qui prévalent, et alors il conviendrait de connaître comment un individu perçoit les opinions de son entourage $^{5}$. Nous supposons plutôt que, en cette matière délicate qu'est la vie sexuelle, personne n'est dupe, chacun admet la possibilité d'un écart entre une norme annoncée et une conduite effective, de sorte que les comportements d'autrui constituent une référence plus significative.

Pour que ces comportements d'autrui soient porteurs d'influence, encore faut-il qu'ils soient connus. Le commerce de la sociabilité permet au mieux de savoir qui "sort" avec qui. Audelà, les activités sont privées et cachées, notamment les pratiques sexuelles qui peuvent faciliter ou prévenir la transmission du VIH. Il faut les dévoiler, en parler, pour qu'elles soient connues. Nous avons donc supposé qu'une analyse des relations dans lesquelles il est possible de parler de questions affectives et sexuelles, si elle ne couvre pas l'ensemble des influences relationnelles, en saisit du moins une partie significative.

En 1985, le National Opinion Research Center de l'Université de Chicago a demandé dans un sondage national quelles sont "les personnes avec lesquelles vous parlez de problèmes importants pour vous ?" (MARSDEN, 1987, BURT, 1985). Cette question permettait d'isoler un noyau de relations proches et particulièrement significatives pour les acteurs. On a utilisé une procédure analogue pour isoler les relations "de confidence"6. Le questionnaire ACSF demandait ainsi "... en dehors de la personne avec qui vous vivez, avec combien de personnes

\footnotetext{
4 Il serait paradoxal, en réponse à une "demande sociale" qui s'interroge sur la possibilité d'infléchir les comportements sexuels, de proposer un cadre interprétatif qui expliquerait la vie sexuelle à partir de déterminismes structuraux peu modifiables. Les postulats retenus ici rendent concevables des changements. .

5 Cette perspective, inscrite dans l'idée des "cercles sociaux", est examinée conjointement dans l'enquête Analyse des Comportements Sexuels en France (ACSF) par A.DEGENNE.

6 Une pré-enquête ( $\mathrm{N}=200)$ avait permis de montrer que cette procédure était possible (FERRAND, MOUNIER, 1990).
} 
discutez-vous d'aventures amoureuses, de problèmes ou de maladies sexuelles, ou de votre vie en couple ?"

Le questionnaire permet de décrire en détail un maximum de trois relations. On s'intéresse d'abord à l'identité du confident (sexe ; âge ; profession), puis aux caractéristiques de la relation (durée; fréquence de contacts; type du lien:ami, parent, collègue; existence éventuelle d'une relation amoureuse) ; enfin à la perception par l'enquêté de quelques dimensions du comportement sexuel de ce confident (nombre de partenaires; changements depuis l'apparition du Sida ; orientation homosexuelle).

Les résultats discutés ici portent sur ce qu'on pourrait nommer "la logique sociale de la confidence". Il s'agit en somme des prolégomènes à une analyse des influences relationnelles : en effet, on ne parle de sa vie personnelle qu'à certaines personnes, et ce qui conditionne ainsi des échanges de parole sur la sexualité, "pré-conditionne" ou interagit avec les influences qui peuvent en résulter. On présentera d'abord quels acteurs développent plus ou moins de relations de confidence. Ensuite on examinera les caractéristiques des relations de discussion, et notamment entre qui des confidences sont échangées. Enfin, dans la mesure où l'acteur pourrait recomposer son réseau pour créer une cohérence normative (réduction de la dissonance), on s'intéressera à l'ancienneté et au renouvellement des relations.

\subsection{Avoir des confidents : une composante spécifique de la sociabilité}

Une première image globale de la confidence dans la société française tient en trois chiffres arrondis : 40\% des personnes n'ont cité aucun confident, $40 \%$ en ont cité deux ou plus de deux, $20 \%$ n'en ont cité qu'un.

TABLEAU 1. - Distribution des enquêtés selon le nombre de confidents

\begin{tabular}{ccccc}
\multicolumn{3}{c}{ Nombre de confidents } \\
$\odot$ & 1 & 2 et plus & \multicolumn{2}{c}{ Ensemble } \\
$\%(1)$ & $\%$ & $\%$ & & Effectif \\
38,7 & 21,6 & 39,7 & $100, \odot$ & 2342
\end{tabular}

Les hommes sont plus nombreux que les femmes à n'avoir aucun confident (46,7\% contre 30,6\%). Passée la soixantaine, les aînés sont quatre fois plus nombreux que les jeunes à n'avoir aucun interlocuteur (60,5\% pour la classe des 60-69 ans contre 16,3\% pour celle des 18-23 ans) ; pour les hommes c'est à partir de quarante ans que l'on observe une baisse sensible dans le nombre de personnes à qui on parle d'affaires affectives et sexuelles. Les femmes ont un régime différent : entre 24 et 29 ans elles réduisent le nombre de leurs confidents, de 30 à 39 ans elles poursuivent dans cette voie pour, à partir de quarante ans, abandonner les échanges verbaux sur les questions affectives et sexuelles mais dans une moindre mesure que les hommes.

Les variations entre milieux socio-professionnels n'étant pas les mêmes pour chaque sexe, il convient d'examiner hommes et femmes séparément (Tableau 2). C'est parmi les hommes appartenant ou ayant appartenu à la catégorie des "cadres et professions intellectuelles supérieures" qu'on trouve la plus forte proportion d'individus réservés (aucun confident, 61,4\%), suivie par celle des "professions intermédiaires" (54\%). Chez les cadres supérieurs, connus par 
ailleurs pour avoir de nombreuses relations personnelles et dont les activités professionnelles sont souvent très sociables, cette faible expansivité est surprenante. Parmi les inactifs la proportion des individus réservés est particulièrement faible, mais ce sont majoritairement des jeunes (66,7\% des inactifs ont moins de 24 ans).

Parmi les femmes, les ouvrières présentent la plus forte proportion de personnes discrètes sur les questions affectives et sexuelles ${ }^{7}$. Parmi les inactives, comme pour les hommes, cette proportion est faible.

Ainsi, à l'exception des inactifs, l'appartenance professionnelle influence de façon très différente la prédisposition à avoir des confidents pour les hommes et pour les femmes. L'écart entre une logique masculine et féminine de la parole est maximum chez les cadres : les femmes y sont moitié moins nombreuses que les hommes à ne pas discuter (32,1\% et 61,4\%). Les employés masculins sont proportionnellement plus nombreux à n'avoir qu'un seul confident ; cette tendance est atténuée chez les femmes employées qui sont aussi les moins nombreuses à ne pas se confier sur les questions affectives et sexuelles.

La liaison entre la propension à échanger sur la vie affective et sexuelle et le fait d'être inséré au moment de l'enquête dans un emploi est plus complexe : l'effet de l'âge se retrouve chez les retraités qui sont majoritairement sans confidents $(60,1 \%$ contre $38,7 \%$ dans l'échantillon) et chez les interviewés poursuivant leurs études qui en ont plusieurs (66,7\%). Les personnes se déclarant au chômage au moment de l'enquête (4,6\% de l'échantillon pondéré) ont des conduites relationnelles en matière de confidence équivalentes à celle de la moyenne de la population enquêtée : globalement le chômage ne semble pas s'accompagner d'une rupture avec ce milieu relationnel très proche que nous explorons. Mais des logiques différentes distinguent les chômeurs et les chômeuses : les premiers déclarent plus de confidents (46,3\% contre 36,9\%) tandis que les femmes, quel que soit leur âge, se caractérisent par une plus grande absence d'échanges sur la vie affective et sexuelle (33,5\% contre 30,6\%). Cet effet est produit par les nouveaux chômeurs (REICHMANN, 1991) ou ceux ayant connu une période de chômage de moins de six mois dans les cinq dernières années: en effet les personnes au chômage aujourd'hui, et ayant connu une période de chômage de plus de six mois dans les cinq dernières années (70\% des chômeurs), déclarent parler de questions affectives et sexuelles tout autant que les autres.

La confidence est une composante de la sociabilité marquée par l'objet même des discussions : la vie sexuelle personnelle. Une autre enquête nationale représentative portant, elle, sur toutes les relations de discussion (HERAN, 1988), permet de relever certains contrastes : l'écart homme/femme, la décroissance avec l'âge, sont plus marqués pour les confidences.

Sur cette toile de fond, le type de vie sexuelle (nombre de partenaires dans les 12 derniers mois : Tableau 3) introduit une forte distinction : les enquêtés multipartenaires sont deux fois moins nombreux que les monopartenaires à n'avoir aucun confident (25,8\% contre 49,8\%) ; les enquêtées trois fois moins nombreuses (11,3\% contre 31,1\%). Neuf femmes multipartenaires sur dix ont au moins un confident. Ainsi la faculté de trouver des interlocuteurs pour parler de sa vie

7 On observe une tendance plus forte chez les femmes "artisans, commerçants, chefs d'entreprise", mais les effectifs sont faibles. 
sexuelle semble aller globalement de pair avec le degré d'activité de l'individu dans ce domaine : l'expansivité relationnelle joue simultanément pour le geste et la parole.

Dans le même sens, 44,5\% des personnes qui vivent en couple ne déclarent aucun confident (autre que le conjoint), contre 25,9\% pour celles qui vivent seules. Cette solitude, purement domiciliaire, favorise même le fait d'avoir au moins deux confidents : 50,5\% contre $34,8 \%$ pour "vit en couple". Mais ceux qui "vivent en couple" pouvaient être désavantagés par l'interdiction de citer le partenaire sexuel stable comme confident. 
TABLEAU 2. - Distribution des enquêtés selon le nombre de confidents cités

$$
\text { Nombre de confidents cités }
$$

\begin{tabular}{|c|c|c|c|c|c|}
\hline \multirow[t]{2}{*}{ Enquêtés } & $\odot$ & 1 & $2+$ & \multicolumn{2}{|c|}{ Ensemble } \\
\hline & $\%$ & $\%$ & $\%$ & \multicolumn{2}{|r|}{ Effectif } \\
\hline Tous & 38,7 & 21,6 & 39,7 & 100,0 & 2342 \\
\hline Hommes & 46,7 & 16,6 & 36,7 & 100,0 & 1303 \\
\hline \multicolumn{6}{|l|}{1303} \\
\hline Femmes & 30,6 & 26,7 & 42,7 & 100,0 & 1039 \\
\hline 18-23 ans & 16,3 & 26,6 & 57,1 & 100,0 & 548 \\
\hline 24-29 ans & 23,0 & 27,3 & 49,7 & 100,0 & 541 \\
\hline $30-39$ ans & 31,4 & 24,4 & 44,2 & 100,0 & 589 \\
\hline $40-49$ ans & 45,1 & 21,2 & 33,7 & 100,0 & 371 \\
\hline $50-59$ ans & 54,6 & 14,6 & 30,8 & 100,0 & 182 \\
\hline $60-69$ ans & $6 \odot, 5$ & 15,6 & 23,9 & 100,0 & 111 \\
\hline \multicolumn{6}{|c|}{ Taille de l'agglomération de résidence } \\
\hline moins de 5000 & 42,4 & 20,0 & 37,6 & 100,0 & 527 \\
\hline 5000 à 50000 & 44,0 & 19,9 & 36,1 & 100,0 & 375 \\
\hline 50000 à 200000 & 40,7 & 20,2 & 39,1 & 100,0 & 314 \\
\hline 200000 et plus & 29,1 & 27,0 & 43,9 & 100,0 & 654 \\
\hline Région Paris & 35,9 & 21,2 & 42,9 & 100,0 & 468 \\
\hline
\end{tabular}

Catégories socio-professionnelles actuelles ou anciennes Enquêtés hommes

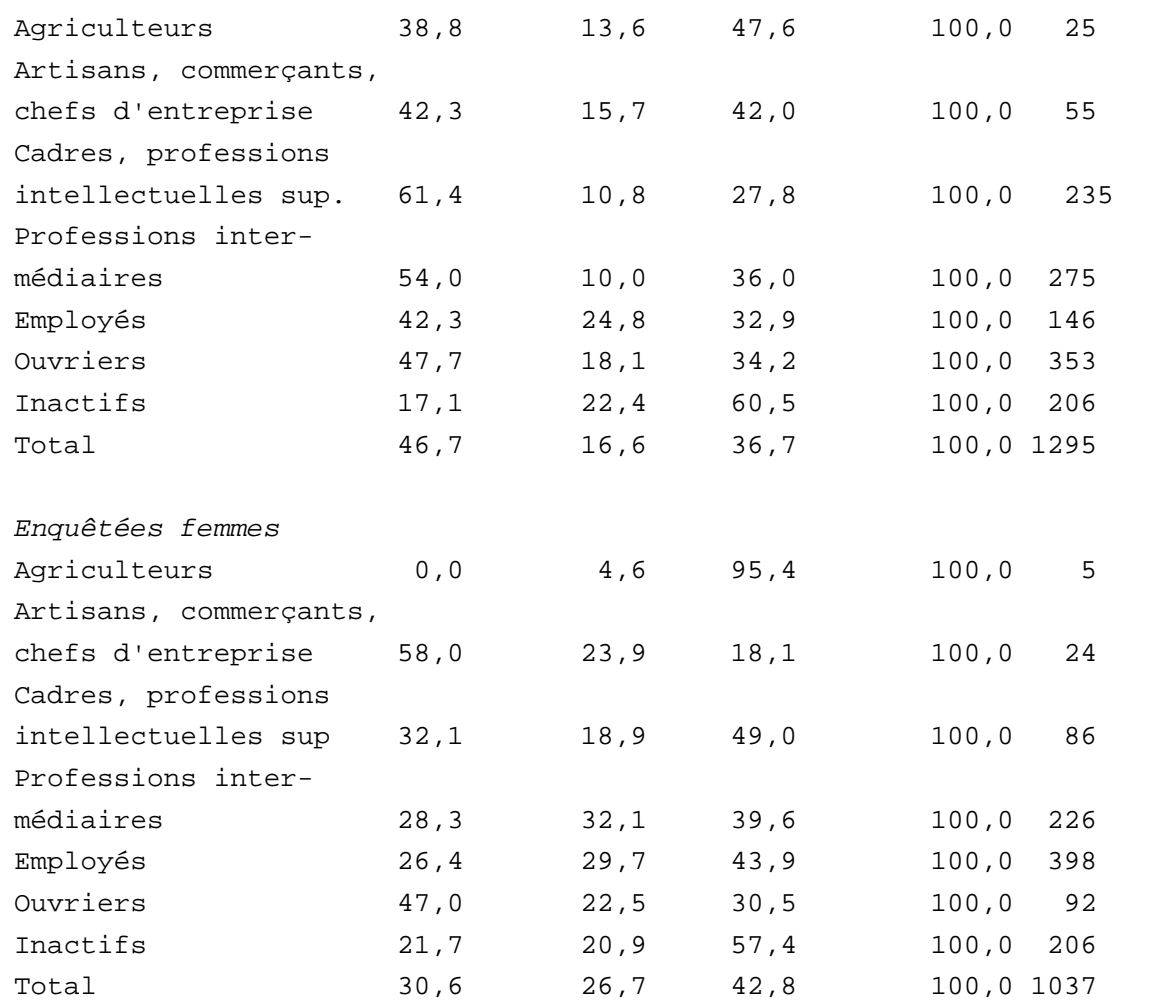


TABLEAU 3. - Distribution des enquêtés selon leur type de vie sexuelle et le nombre de confidents cités

\begin{tabular}{|c|c|c|c|c|c|}
\hline \multirow{3}{*}{ Enquêtés } & \multicolumn{3}{|c|}{ Nombre de confidents cités } & \multirow{2}{*}{\multicolumn{2}{|c|}{ Ensemble }} \\
\hline & $\odot$ & 1 & $2+$ & & \\
\hline & $\%$ & $\%$ & $\%$ & & Effectif \\
\hline \multicolumn{6}{|l|}{ Mode de vie } \\
\hline vivent en couple & 44,5 & 20,7 & 34,8 & $10 \odot, 0$ & 1164 \\
\hline hommes & 52,3 & 15,4 & 32,3 & $10 \odot, 0$ & 652 \\
\hline femmes & 35,9 & 26,5 & 37,6 & $10 \odot, \odot$ & 512 \\
\hline vivent seuls & 25,9 & 23,6 & $5 \odot, 5$ & $10 \odot, \odot$ & 1178 \\
\hline hommes & 32,7 & 19,6 & 47,7 & $10 \odot, \odot$ & 651 \\
\hline femmes & 19,9 & 27,2 & 52,9 & $100, \odot$ & 527 \\
\hline
\end{tabular}

Nombre de partenaires sexuels en 12 mois : Hommes

$\begin{array}{lrrrrr}\odot \text { partenaire } & 61,4 & 6,1 & 32,5 & 100, \odot & 35 \\ \text { monopartenaire } & 49,8 & 16,3 & 33,8 & 100, \odot & 686 \\ \text { multipartenaire } & 25,8 & 19,2 & 55, \odot & 100, \odot & 566 \\ \text { Tous } & 46,9 & 16,4 & 36,7 & 100, \odot & 1287 \\ & & & & & \\ \text { Femmes } & & & & & \\ \begin{array}{l}\odot \text { partenaire } \\ \text { monopartenaire }\end{array} & 32,6 & 12,8 & 38,7 & 100, \odot & 64 \\ \text { multipartenaire } & 11,3 & 28,9 & 40, \odot & 100, \odot & 626 \\ \text { Toutes } & 30,8 & 23,1 & 65,6 & 100, \odot & 297 \\ & & 27,8 & 41,5 & 100, \odot & 987\end{array}$

\subsection{Nature des relations et composition des réseaux de confidence}

Le questionnaire permettait à chaque enquêté de décrire en détail un maximum de trois relations de confidence : les caractéristiques de 3546 relations ont ainsi été recueillies.

\subsubsection{LES LIENS QUI AUTORISENT LA CONFIDENCE}

Les relations de confidences pouvaient être définies par la nature du lien : amical, de travail, de parenté. Nature éminemment sociale: en qualifiant un lien par l'une des trois catégories proposées, les enquêtés sont supposés se référer au modèle social (le "rôle") qui définit le mieux cette relation ${ }^{8}$. On analysera ensuite comment la composition des réseaux varie selon quelques caractéristiques socio-démographiques des enquêtés qui citent des relations.

TABLEAU 4. - Distribution des relations de confidence selon la nature du lien par statut d'activité des enquêtés

\begin{tabular}{|c|c|c|c|cc|}
\hline $\begin{array}{c}|c| \\
\text { Relations citées } \\
\text { Par }\end{array}$ & \multicolumn{3}{|c|}{ Nature du lien } & Tous liens \\
\hline & Collègue & Famille & Amis & & \\
\hline Ensemble & 13,8 & 24,2 & 62,0 & 100 & 3546 \\
\hline Actif et ancien actif & 16,4 & 24,9 & 58,7 & 100 & 2761 \\
\hline Inactif & 1,9 & 20,8 & 77,3 & 100 & 785 \\
\hline
\end{tabular}

\footnotetext{
8 Les relations sont souvent "polyvalentes" ou "polycontextuelles" : un collègue peut être aussi un ami, etc. Les contraintes de l'enquête ont obligé à imposer le choix d'un modèle unique.
} 
Parmi les différents types de lien autorisant des discussions sur la vie affective et sexuelle, l'amitié prédomine largement. Les relations dans la parenté occupent une place sensiblement identique à celle indiquée dans l'enquête "Contacts entre les personnes" et les collègues sont moins fréquents parmi les confidents que parmi les relations de discussion "tout venant". Les inactifs, par principe dépourvus de collègues comme confidents potentiels, ne se reportent pas sur l'autre pôle formel de la sociabilité : la parenté. Trois quarts des relations qu'ils citent sont amicales.

On parle sexualité dans des relations qui souvent existent par ailleurs et pour d'autres propos. Mais on remarque à nouveau que la confidence s'inscrit dans la sociabilité de façon sélective : certains types de liens découragent les discussions affectives ${ }^{9}$; d'autres semblent au contraire soit les favoriser, soit même exister pour les permettre. Dans l'"amitié", l'importance des affinités réciproques, le caractère privé du lien peu contraint par des rôles sociaux institutionnalisés, sont des caractéristiques formelles de la relation en concordance avec l'objet de la discussion: la vie affective et sexuelle personnelle, dans ses dimensions aussi bien "officielles" que plus souterraines ${ }^{10}$.

\subsubsection{COMMENT LES INDIVIDUS COMPOSENT LEUR RESEAU}

En regroupant les relations citées par certaines catégories d'individus, on en décrit le réseau moyen. En deçà des tendances globales que nous venons d'évoquer, ce sont des logiques sociales particulières de la confidence qui sont mises en lumière. Pour cette analyse on ne prend en compte par la suite que les relations citées par des enquêtés ayant déclaré avoir (ou avoir eu) une activité professionnelle, afin de pouvoir traiter de manière homogène le travail comme contexte possible d'une relation de confidence ${ }^{11}$.

TABLEAU 5. - Distribution des relations de confidence selon le sexe des enquêtés (actifs ou anciens actifs)

\begin{tabular}{|c|c|c|c|r|c|}
\hline Citeurs & \multicolumn{3}{|c|}{ Types de relation } & \multicolumn{2}{r|}{ Ensemble } \\
\hline & Collègue & Famille & Ami & $\%$ & Effectif \\
\hline Ensemble & 16 & 25 & 59 & 100 & 2761 \\
\hline Hommes & 22 & 18 & 60 & 100 & 1457 \\
\hline Femmes & 12 & 31 & 57 & 100 & 1304 \\
\hline
\end{tabular}

Les femmes parlent plus volontiers avec des membres de la parenté, et cette inclination familialiste de la sociabilité féminine est connue. Mais la particularité de la confidence semble être marquée pour elles par le poids important des échanges amicaux, inhabituellement proche ici de celui des hommes.

\footnotetext{
9 Dans la pré-enquête spécifique, on a vu par exemple que les relations de voisinage ou celles nouées dans les associations sont très rarement citées (FERRAND, MOUNIER, 1990).

10 BIDART (Claire), LASMAS-CERCOM, réalise une recherche qualitative soutenue par l'ANRS pour comprendre la dynamique de la confidence dans l'ensemble des relations interpersonnelles d'un individu.

11 Les relations citées par des actifs ou anciens actifs représentent 82\% de l'ensemble. Celles non qualifiées par le travail, la famille ou l'amitié ("autre" et "non-réponses" 1,3\%) ont été agrégées aux relations d'amitié car elles présentent des caractéristiques semblables sur différents items.
} 
Si les plaisanteries sexuelles émaillent parfois les échanges dans la vie de travail, des discussions qui engagent la vie privée de l'individu lui-même ne trouvent pas là un terrain privilégié. L'immixtion d'une parole sur la sexualité dans la sphère du travail semble nettement plus légitime pour les hommes : ils sont deux fois plus nombreux à discuter de questions personnelles avec des collègues que les femmes. Cette différenciation sexuée de la circulation de la parole dans la sphère professionnelle peut relever d'une sociabilité distincte au travail (LAZEGA, 1992) ou bien d'une attitude globale de réserve qui évite de prêter le flanc à d'éventuelles avances insistantes de collègues ou supérieurs.

La répartition des relations en fonction de la catégorie socioprofessionnelle du répondant va dans le sens des résultats des travaux sur la sociabilité : les ouvriers discutent un peu plus des questions affectives et sexuelles avec leur entourage professionnel (surtout les ouvrières) ; les employés privilégient les relations familiales; les cadres et professions intellectuelles supérieures s'adressent davantage aux amis.

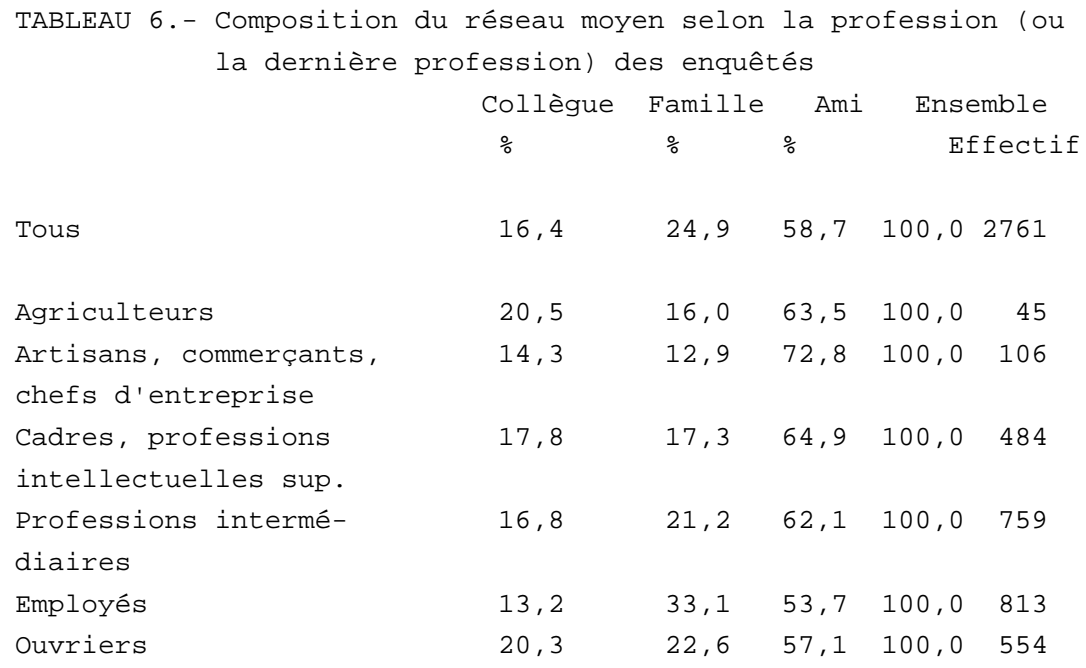

Alors qu'avec l'avance en âge le nombre moyen de confidents diminue de moitié, la composition des réseaux, les processus de sélection des relations indiquent des tendances contrastées. La proportion d'amis reste globalement stable (autour de 58\%), puis augmente à partir de 60 ans. Les changements affectent surtout les proportions de relations familiales qui décroissent (de 36\% à 12,9\%), pendant que la part des relations avec des collègues suit le cycle de l'investissement dans la vie professionnelle : faible avant 24 ans (6,2\%), elle monte jusqu'à 50 ans (pour atteindre 20\%), puis baisse ensuite. Cette distance prise d'avec les relations professionnelles, conduit à privilégier les relations affinitaires amicales plutôt que la parenté. 
TABLEAU 7. - Composition du réseau moyen selon l'âge des enquêtés actifs

actifs et anciens

\begin{tabular}{lccccc} 
& $\begin{array}{c}\text { Collègue } \\
\%\end{array}$ & $\begin{array}{c}\text { Famille } \\
\%\end{array}$ & $\begin{array}{c}\text { Ami } \\
\%\end{array}$ & \multicolumn{2}{c}{ Ensemble } \\
Tous & 16,4 & 24,9 & 58,7 & $100, \odot$ & 2761 \\
& & & & & \\
$18-23$ ans & 6,2 & $36, \odot$ & 57,2 & $10 \odot, \odot$ & 363 \\
$24-29$ ans & 12,1 & 29,9 & $58, \odot$ & $10 \odot, \odot$ & $80 \odot$ \\
$3 \odot-39$ ans & 19,4 & 23,7 & 56,9 & $10 \odot, \odot$ & $9 \odot 2$ \\
$4 \odot-49$ ans & $2 \odot, \odot$ & 21,6 & 58,4 & $10 \odot, \odot$ & $45 \odot$ \\
$5 \odot-59$ ans & $18, \odot$ & 26,6 & 55,4 & $10 \odot, \odot$ & 159 \\
$6 \odot-69$ ans & 14,7 & 12,9 & 72,9 & $10 \odot, \odot$ & 87
\end{tabular}

\subsection{La sélectivité sociale des relations de confidence}

Les individus se confient plus ou moins, et ils le font à travers différents types de liens. Une troisième dimension importante de la logique sociale de la confidence que nous avons à examiner est constituée par les processus qui permettent qu'une relation s'établisse entre certains acteurs et pas entre d'autres. Pour ces échanges très personnels, comme pour le domaine voisin de l'amitié, ou celui plus institutionnalisé du "choix du conjoint", on peut s'attendre à ce qu'une relation ait plus de chances d'exister entre des personnes ayant des statuts biographiques et sociaux peu différents: la proximité sociale facilite souvent ces relations (ROGERS, BHOWMIK, 1971).

Ce principe très général (homophilie) peut, selon les enjeux et les contenus des relations, subir des inflexions particulières : par exemple, des relations comportant une orientation à dominante utilitaire diffèrent d'une relation à dominante affective. Qu'en est-il de la confidence ? Entre quels types d'acteurs ces échanges s'établissent-ils de façon privilégiée ? Différentes voies se présentent pour examiner la sélectivité sociale des relations de confidence.

D'une part, il est possible de ne mettre l'accent que sur les préférences parmi ceux qui se confient : on compare alors les choix effectués par une catégorie particulière de citeurs à la distribution moyenne des choix pour l'ensemble de la population des citeurs ${ }^{12}$. Cette méthode se donne pour modèle de référence la distribution des confidents cités : elle suppose que n'importe quel enquêté aurait la même probabilité de citer tel ou tel type de confident, et met en lumière quels types de confidents sont privilégiés ou au contraire délaissés par une certaine catégorie de citeurs $^{13}$.

D'autre part, il est possible de mettre l'accent sur le processus d'émergence des confidences entre les différentes catégories qui composent la population. Ce processus comporte le fait qu'aucun, peu ou beaucoup de liens s'établissent entre deux catégories de personnes. Au lieu de prendre pour référence la confidence telle qu'elle existe entre les acteurs qui ont cité des confidents, pour n'examiner que des distorsions internes, elle suppose qu'une relation pourrait se

\footnotetext{
12 Méthode employée par exemple dans la présentation de certains résultats de l'enquête INED sur le choix du conjoint (BOZON, HERAN, 1987).

13 Dans cet esprit indiquons que 74,1\% des relations sont établies entre personnes de même sexe ; 56,9\% entre personnes appartenant à la même classe d'âge décennale ; 39,2\% à la même catégorie socio-professionnelle.
} 
former entre des individus de n'importe quelle catégorie, à proportion du poids de ces catégories dans la population globale : les distributions moyennes utilisées comme références sont celles des individus comme termes potentiels des relations. On compare ensuite les probabilités constatées aux probabilités théoriques par case.

Cette seconde comparaison conduit les relations inexistantes, celles qui sont prohibées par la différence de sexe, par des écarts d'âge ou par la distance sociale à peser autant que les liens existants et à leur donner d'autant plus de relief. Par ailleurs elle ne neutralise pas les différences d'expansivité relationnelle: les catégories qui citent davantage de confidents peuvent, dans la structure globale de la matrice, à la fois se sursélectionner elles-mêmes et relativement peu sous- sélectionner d'autres catégories. Le jeu relationnel est interdit à celles qui citent globalement moins de confidents que la moyenne ${ }^{14}$. Enregistrer cet effet est important car on sait que, passé un seuil minimal, l'accroissement du nombre de relations va souvent de pair avec leur diversification : dans le cas examiné ici, même avec une faible variation autorisée du nombre de liens (de 0 à 3), l'existence de contacts avec des catégories un peu et très différentes est relativement facilitée par un expansionnisme relationnel plus fort. Enfin cette analyse décrit évidemment les choix socialement préférentiels, mais on peut la lire également comme décrivant la conséquence de ces choix sur des flux d'informations ou d'influence traversant ou non le champ social. Une catégorie qui cite particulièrement peu de confident apparaîtra pour ce qu'elle est : à la fois particulièrement "débranchée" des autres catégories et avec peu de communications internes. Dans cette lecture, l'expansivité relationnelle ouvre la voie à des flux qui apparaîtront relativement plus puissants ou plus largement distribués dans l'espace social ${ }^{15}$.

On analysera donc deux processus de distribution sociale de la confidence :

- la déformation globale de l'espace des choix, telle qu'elle résulte de la comparaison des distributions marginales théoriques et observées ;

- la force des processus d'auto-sélection selon les catégories ;

\footnotetext{
14 On comprend par ces remarques que la logique d'analyse ne peut être la même que pour le choix du conjoint en société monogame : ici chacun peut citer plusieurs confidents ou aucun et être cité plusieurs fois ou jamais. Par contre, la question évoquée ici est homologue à celle posée par les relations sexuelles existant durant un intervalle donné de temps, lorsqu'on tient compte du multipartenariat.

15 Dans l'ensemble de la matrice, la somme algébrique des écarts entre le modèle et l'observé vaut zéro : toute relation "manquante" est, quelque part dans la matrice, compensée par une relation "surexistante".
} 
TABLEAU 8. - Homophilie et hétérophilie des relations de confidence

8a) Effet de l'âge

Relations non-pondérées : 3546

\begin{tabular}{|c|c|c|c|c|c|c|c|c|}
\hline & Réce & oteurs & des rel & ations & & & & \\
\hline & $18-23$ & $24-29$ & $30-39$ & $40-49$ & $50-59$ & $60+$ & $\begin{array}{l}\text { expan- } \\
\text { sivité }\end{array}$ & $\begin{array}{l}\text { indice d' } \\
\text { homo- }\end{array}$ \\
\hline prob obs E & 21,0 & 16,7 & 24,3 & 17,4 & 11,7 & 8,9 & $E-T$ & philie \\
\hline prob théo $\mathrm{T}$ & 14,7 & 13,3 & 21,7 & 19,6 & 16,4 & 14,3 & & (obs/théo) \\
\hline prob obs $\mathrm{R}$ & 17,6 & 16,9 & 24,3 & 20,4 & 11,0 & 9,8 & & \\
\hline attractivité R-théo & $+2,9$ & $+3,6$ & $+2,6$ & $+\odot, 8$ & $-5,4$ & $-4,5$ & & \\
\hline Emetteurs & & & & & & & & \\
\hline $18-23$ & & & & & & & $+6,3$ & 6,5 \\
\hline prob théo & 2,2 & 2,0 & 3,2 & 2,9 & 2,4 & 2,1 & & \\
\hline prob obs & 14,2 & 2,9 & 1,1 & 1,7 & $\odot, 9$ & $\odot, 1$ & & \\
\hline obs-théo & +12 & $+\odot, 9$ & $-2,1$ & $-1,2$ & $-1,5$ & $-2,0$ & & \\
\hline $24-29$ & & & & & & & $+3,4$ & 4,6 \\
\hline prob théo & 2,0 & 1,8 & 2,9 & 2,6 & 2,2 & 1,9 & & \\
\hline prob obs & 2,0 & 8,3 & 3,3 & 1,6 & 1,2 & $\odot, 3$ & & \\
\hline obs - théo & & $+6,5$ & $+\odot, 4$ & $-1,0$ & $-1,0$ & $-1,6$ & & \\
\hline $30-39$ & & & & & & & $+2,6$ & 2,8 \\
\hline prob théo & 3,2 & 2,9 & 4,7 & 4,2 & 3,6 & 3,1 & & \\
\hline prob obs & 0,9 & 4,1 & 13,2 & 4,0 & $\odot, 8$ & 1,2 & & \\
\hline obs - théo & $-2,3$ & $+1,2$ & $+8,5$ & $-\odot, 2$ & $-2,8$ & $-1,9$ & & \\
\hline $40-49$ & & & & & & & $-2,2$ & 2,1 \\
\hline prob théo & 2,9 & 2,6 & 4,2 & 3,8 & 3,2 & 2,8 & & \\
\hline prob obs & 0,3 & $\odot, 7$ & 4,8 & 8,2 & 2,1 & 1,3 & & \\
\hline obs-théo & $-2,6$ & $-1,9$ & $+2,6$ & $+4,4$ & $-1,1$ & $-1,5$ & & \\
\hline $50-59$ & & & & & & & $-4,7$ & 1,7 \\
\hline prob théo & 2,4 & 2,2 & 3,6 & 3,2 & 2,7 & 2,3 & & \\
\hline prob obs & & $\odot, 9$ & 1,6 & 3,1 & 4,6 & 1,6 & & \\
\hline obs-théo & $-2,4$ & $-1,3$ & $-2,0$ & $-0,1$ & $+1,9$ & $-\odot, 7$ & & \\
\hline $60-69$ & & & & & & & $-5,4$ & 2,7 \\
\hline prob théo & 2,1 & 1,9 & 3,1 & 2,8 & 2,3 & 2,0 & & \\
\hline prob obs & 0,1 & $\odot, 1$ & $\odot, 3$ & 1,7 & 1,5 & 5,3 & & \\
\hline obs-théo & $-2,0$ & $-1,8$ & $-2,8$ & $-1,1$ & $-\odot, 8$ & $+3,3$ & & \\
\hline
\end{tabular}


8b) Effet de la catégorie socio-professionnelle

Relations non-pondérées : 3534

\begin{tabular}{|c|c|c|c|c|c|c|c|c|c|c|}
\hline & \multicolumn{4}{|c|}{ Récepteurs des relations } & \multirow[b]{2}{*}{5} & \multirow[b]{2}{*}{6} & \multirow[b]{2}{*}{7} & \multirow[b]{2}{*}{8} & \multirow[b]{2}{*}{$\begin{array}{l}\text { expan- } \\
\text { sivité }\end{array}$} & \multirow[b]{2}{*}{$\begin{array}{l}\text { indice } \\
\text { d'homo }\end{array}$} \\
\hline & 1 & 2 & 3 & 4 & & & & & & \\
\hline prob obs $E$ & 1,1 & 3,6 & 8,2 & 13,6 & 29,9 & 16,7 & 9,4 & 17,5 & $E-T$ & philie \\
\hline prob théo $\mathrm{T}$ & 1,4 & 3,9 & 8,3 & 13,1 & 25,9 & 20,1 & 15,0 & 12,2 & & obs/théo \\
\hline prob obs $\mathrm{R}$ & 1,3 & 4,8 & 10,3 & 13,8 & 26,3 & 9,4 & 8,3 & 25,8 & & \\
\hline attractivité $\mathrm{R}$-théo & $-\odot, 9$ & $+\odot, 9$ & $+2,0$ & $+\odot, 7$ & $+\odot, 4$ & $-10,7$ & $-6,7$ & $+13,6$ & & \\
\hline \multicolumn{11}{|l|}{ Emetteurs } \\
\hline 1 agriculteurs & & & & & & & & & $-\odot, 3$ & \\
\hline prob théo & & $\odot, 1$ & $\odot, 1$ & $\odot, 2$ & $\odot, 4$ & $\odot, 3$ & $\odot, 2$ & $\odot, 2$ & & \\
\hline prob obs & $\odot, 2$ & $\odot, 1$ & $\odot, 1$ & & $\odot, 4$ & $\odot, 1$ & $\odot, 1$ & $\odot, 1$ & & \\
\hline obs-théo & $+\odot, 2$ & & & $-\odot, 2$ & & $-\odot, 2$ & $-\odot, 1$ & $-0,1$ & & \\
\hline \multicolumn{11}{|l|}{2 artisans com. } \\
\hline chefs d'entreprise & & & & & & & & & $-\odot, 3$ & 8 \\
\hline prob théo & 0,1 & $\odot, 1$ & $\odot, 3$ & $\odot, 5$ & 1,0 & $\odot, 8$ & 0,6 & 0,5 & & \\
\hline prob obs & & $\odot, 8$ & $\odot, 6$ & $\odot, 3$ & 1,0 & $\odot, 1$ & 0,1 & $\odot, 6$ & & \\
\hline obs-théo & $-\odot, 1$ & $+\odot, 7$ & $+\odot, 3$ & $-\odot, 2$ & & $-\odot, 7$ & $-\odot, 5$ & $+\odot, 1$ & & \\
\hline 3 cadres prof. & & & & & & & & & & 4,3 \\
\hline intel. sup. & & & & & & & & & $-0,1$ & \\
\hline prob théo & 0,1 & $\odot, 3$ & $\odot, 7$ & 1,1 & 2,1 & 1,7 & 1,2 & 1,0 & & \\
\hline prob obs & & $\odot, 5$ & $3, \odot$ & 2,2 & 1,1 & $\odot, 1$ & 0,4 & $\odot, 9$ & & \\
\hline obs-théo & $-\odot, 1$ & $+\odot, 2$ & $+2,3$ & $+1,1$ & $-1,0$ & $-1,6$ & $-\odot, 8$ & $-0,1$ & & \\
\hline \multicolumn{11}{|l|}{4 professions } \\
\hline intermédiaires & & & & & & & & & $+\odot, 5$ & 2,4 \\
\hline prob théo & $\odot, 2$ & $\odot, 5$ & 1,1 & 1,8 & 3,4 & 2,6 & 2,0 & 1,6 & & \\
\hline prob obs & 0,1 & $\odot, 7$ & 1,5 & 4,3 & 3,9 & $\odot, 4$ & 0,3 & 2,2 & & \\
\hline obs-théo & $-0,1$ & $+\odot, 2$ & $+\odot, 4$ & $+2,5$ & $+\odot, 5$ & $-2,2$ & $-1,7$ & $+\oplus, 6$ & & \\
\hline 5 employés & & & & & & & & & +4 & 1,8 \\
\hline prob théo & $\odot, 4$ & 1,0 & 2,1 & 3,4 & 6,7 & 5,2 & 3,9 & 3,1 & & \\
\hline prob obs & 0,1 & 1,3 & 2,4 & 3,4 & 11,9 & 2,3 & 2,8 & 5,7 & & \\
\hline obs-théo & $-0,3$ & $+\odot, 2$ & $+\odot, 3$ & & $+5,2$ & $-2,9$ & $-1,1$ & $+2,6$ & & \\
\hline 6 ouvriers & & & & & & & & & $-3,4$ & 1,3 \\
\hline prob théo & $\odot, 3$ & $\odot, 8$ & 1,7 & 2,6 & 5,2 & 4,0 & 3,0 & 2,5 & & \\
\hline prob obs & 0,1 & 1,2 & $\odot, 9$ & 1,0 & 4,2 & 5,1 & 0,5 & 3,7 & & \\
\hline obs-théo & $-0,2$ & $+\odot, 4$ & $-\odot, 8$ & $-1,6$ & $-1,0$ & $+1,1$ & $-2,5$ & $5+1,2$ & & \\
\hline 7 retraités & & & & & & & & & $-5,6$ & 1,6 \\
\hline prob théo & $\odot, 2$ & $\odot, 6$ & 1,2 & 2,0 & 3,9 & 3,0 & 2,3 & 1,8 & & \\
\hline prob obs & $\odot, 6$ & & $\odot, 5$ & $\odot, 8$ & 1,4 & $\odot, 6$ & 3,6 & 1,9 & & \\
\hline obs-théo & $+\Theta, 4$ & $-\odot, 6$ & $-\odot, 7$ & $-1,2$ & $-2,5$ & $-2,4$ & $+1,3$ & $+0,1$ & & \\
\hline 8 non-actifs & & & & & & & & & $+5,3$ & 7,1 \\
\hline prob théo & $\odot, 2$ & $\odot, 5$ & 1,0 & 1,6 & 3,1 & 2,5 & 1,8 & 1,4 & & \\
\hline prob obs & 0,1 & 0,3 & 1,3 & 1,7 & 2,4 & $\odot, 7$ & 0,5 & 10,7 & & \\
\hline obs-théo & $-0,1$ & $-\odot, 2$ & $+\odot, 3$ & $+\Theta, 1$ & $-\odot, 7$ & $-1,8$ & $-1,3$ & $3+9,3$ & & \\
\hline
\end{tabular}




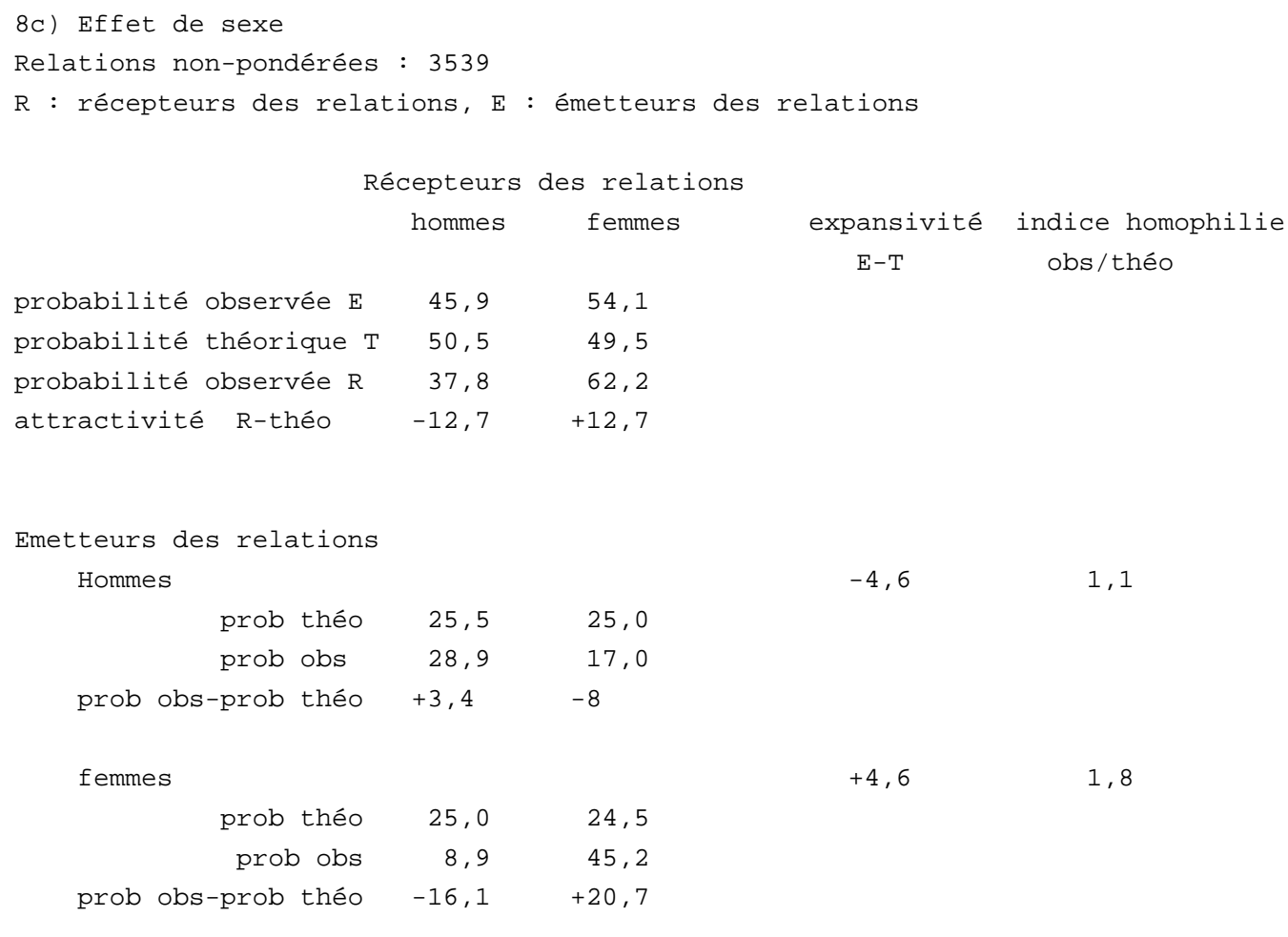

$$
-4,6 \quad 1,1
$$$$
\text { + }
$$

\subsubsection{LA BARRIERE DES AGES (TABLEAU 8A)}

Les écarts des totaux marginaux de lignes et de colonnes indiquent respectivement l'expansivité et l'attractivité relationnelle relative de chaque catégorie. Ils décroissent avec l'âge et deviennent négatifs les uns à partir de 40 ans (on cite moins de confidents) et les autres à partir de 50 ans (on est moins cité comme confident) : ainsi on "résiste" mieux à l'avance en âge comme récepteur de confidence que comme personne citant des confidents. Mais, passé la cinquantaine, l'espace social de la confidence est globalement "dépressif".

Sur l'ensemble du tableau, la somme des écarts positifs avoisine 40\% : deux relations sur dix résultent d'une sursélection entre catégories d'âge ${ }^{16}$.

A tous âges, les confidences s'échangent de façon privilégiée avec des personnes d'âges proches. Avant 24 ans on trouve six fois plus de relations homophiles que ne le demanderait une modèle d'équirépartition. Cette pression homophile diminue ensuite de manière régulière jusqu'à 60 ans, où le ratio tombe à 1,7 pour remonter ensuite à $2,7{ }^{17}$. Mais cette puissance des choix homophiles aux âges jeunes ne signifie pas que ces populations s'enfermeraient dans un "ghetto" de la parole sur la vie affective et sexuelle.

\footnotetext{
16 En d'autres termes, il faudrait permuter les partenaires de deux relations sur dix pour rétablir entre chaque paire de catégories un flux d'échange "équitable", proportionnel à leur poids dans l'ensemble de la population.

17 Probabilité constatée divisée par la probabilité attendue. Cf Tableau 8 dernière colonne.
} 
En effet, l'homophilie résulte de deux processus : une auto-sélection forte et/ou une soussélection des "autres". Laquelle de ces logiques prédomine ${ }^{18}$ ? Avant 30 ans on se surchoisit deux fois plus qu'on ne souschoisit d'autres catégories ; entre 50 et 59 ans on se surchoisit trois fois moins qu'on ne souschoisit les autres catégories : dans les âges jeunes l'expansivité relationnelle permet d'être en contact avec différents groupes, et "en plus" énormément avec son semblable ; plus tard, l'auto-centrage de la parole résulte massivement d'un déficit d'ouverture aux autres catégories. Ainsi deux profils typiques peuvent être opposés : des confidences jeunes qui sont homophiles par "excès", des confidences âgées qui sont homophiles par "défaut".

\subsubsection{LES NIVEAUX SOCIAUX (TABLEAU 8B)}

Une évaluation de l'homophilie selon les milieux sociaux doit tenir compte du caractère rudimentaire des informations qu'il est possible de demander à un enquêté pour décrire un confident : on ne peut notamment pas savoir l'ancienne activité des retraités. Cette catégorie, et celle des "inactifs" majoritairement en cours d'étude, incorporent de plus, un effet d'âge, aux deux bornes du cours de la vie.

Globalement la comparaison entre la distribution des enquêtés et celle des confidents fait ressortir cet effet (surcitation des inactifs "jeunes" et sous-citation des retraités "âgés") et un seul trait significatif en termes de milieu social : la sous-citation massive des "ouvriers". Phénomène qui prend tout son relief lorsque les autres catégories d'actifs apparaissent, elles, citées sensiblement à proportion de leur représentation dans la population.

Au total, trois relations sur dix sont décalées par rapport à une répartition égalitaire des confidences. Mais les clivages socio-professionnels de la confidence sont moins marqués que ceux des âges ${ }^{19}$ : ainsi les échanges de paroles sur la vie affective et sexuelle sont moins sensibles à des différences d'appartenances socio-économiques qu'à des différences d'avance en âge. Si l'on suppose que les discussions demandent une proximité normative, langagière, il est logique que les "sous-cultures" de la sexualité soient d'abord marquées par l'âge, qui peut aussi se comprendre comme un effet de génération.

La hiérarchie sociale différencie le degré de sélectivité des relations de confidence : les "artisans commerçants, chefs d'entreprise" se citent eux-même 8 fois plus que leur poids dans la population globale, les ouvriers seulement 1,3 fois. Un décrochement net sépare les "professions libérales et cadres supérieurs" (4,3) des "professions intermédiaires" $(2,4)$. Les échanges de paroles sur la sexualité et la vie affective apparaissent ainsi sensibles aux écarts sociaux : "en haut de l'échelle" la confidence privilégie les pairs ; les "intermédiaires" et les "employés" font preuve de plus d'ouverture et d'éclectisme. Mais pas forcément pour sélectionner des confidents plus "prestigieux" que soi : les "intermédiaires" sursélectionnent presqu'autant des employés $(1,1)$ que des cadres $(1,4)$.

\footnotetext{
18 On utilise un indicateur simple : le ratio par catégorie entre somme des écarts négatifs (sous-sélection) et somme des écarts positifs (la case homophile, et parfois une autre case).

19 La matrice des choix professionnels a 64 cases, contre 36 pour les âges : la somme des écarts pouvait, à intensité égale de la sursélection, être plus importante.
} 


\subsubsection{LES CONFIDENTS SONT DES CONFIDENTES (TABLEAU 8C)}

Les relations de confidences s'établissent de manière privilégiée avec des femmes : on trouve un écart de 12,7 points entre la proportion des femmes enquêtées (49,5\%) et la proportion des confidentes (62,2\%. Symétriquement les confidents sont sous-représentés d'autant). Cette "popularité" plus grande des femmes s'accompagne aussi d'une plus grande expansivité : on trouve 4,6\% de relations "en trop" citées par des femmes.

La somme des écarts est de 24 : il faudrait permuter les partenaires de deux relations sur dix pour rétablir un espace homogène de la confidence.

Les femmes choisissent 1,8 fois plus souvent un confident de même sexe, alors que le rapport n'est que de 1,1 pour les hommes. Et globalement elles ont des choix plus marqués que ceux des hommes: les écarts entre probabilités constatées et attendues sont beaucoup plus importants que pour les hommes. Notamment la moindre citation "hétérosexuelle" est deux fois plus importante chez les femmes que chez les hommes. Si les confidents sont très souvent des confidentes, c'est à la fois parce que les femmes se choisissent plus elles-mêmes et parce qu'elles apprécient fort peu de se confier à une homme.

\subsection{Dynamique des relations amicales}

Ces relations qui autorisent la confidence ne forment pas un univers stable et figé. Elles naissent, durent plus ou moins, et disparaissent du réseau des individus. Dans cet intervalle, bien des choses se passent, et surtout des choses très variées. Nous postulons qu'il existe différentes "formes temporelles" des liens interpersonnels : la nature des échanges entre les partenaires peut évoluer, lentement ou bien par saut qualitatif ou rester stable. Et ces formes des processus relationnels dépendent du degré plus ou moins socialisé ou interpersonnel de régulation des échanges (des liens très inscrits dans un cadre social sont plus stables : FERRAND, 1989a).

Cette dynamique "naturelle" des relations est importante à connaître pour notre propos : voulant évaluer l'influence des confidents, nous devons comprendre si et comment certains acteurs opèrent des recompositions, des manipulations, de leur réseau pour créer une cohérence, un consensus normatif. Le premier pas présenté ici n'examine que des tendances globales, à partir de la simple indication de l'ancienneté de constitution des relations décrites dans le questionnaire. Cette analyse est complétée par des enseignements tirés de l'approche qualitative réalisée par C.BIDART.

Globalement, l'ancienneté moyenne des différents types de liens est contrastée ${ }^{20}$. Pour repérer les logiques différenciées de stabilité et de renouvellement (FISCHER, 1982), nous ne retiendrons qu'un type de lien : les relations amicales dont la part est sensiblement la même à tous les âges (effectif non pondéré : 2406, soit 1812 relations citées par des actifs et anciens actifs et 594 relations citées par des non actifs ; elles représentent 67,8\% de l'ensemble des relations).

\footnotetext{
20 N'examinant que des liens actifs au temps d'enquête, nous ne connaissons pas des "durées de vie" moyennes : le terme d'ancienneté est donc préférable. D'autre part, contrairement à beaucoup de collègues anglo-saxons, nous considérons qu'il est inconsistant de demander "depuis combien de temps on connaît" une personne apparentée.
} 


\subsubsection{L'ANCIENNETE DES LIENS AMICAUX}

L'ancienneté des relations peut être comprise comme résultant de dispositions personnelles des individus à faire durer leurs liens (MATTHEWS, 1986), de contraintes externes (mobilité...), ou enfin de modèles sociaux particuliers.

\begin{tabular}{|c|c|c|c|}
\hline & Effectif & $\begin{array}{c}\text { Ancienneté } \\
\text { moyenne } \\
\text { (en années) }\end{array}$ & $\begin{array}{l}\text { Coefficient } \\
\text { de variation } \\
\text { (écart type/moyenne) }\end{array}$ \\
\hline Toutes relations & 2406 & 10,1 & 86,5 \\
\hline \multicolumn{4}{|c|}{ Par sexe de l'enquêté } \\
\hline homme & 1290 & 10,4 & 82,9 \\
\hline femme & 1116 & 9,8 & 90,8 \\
\hline \multicolumn{4}{|c|}{ Par âge de l'enquêté } \\
\hline $18-23$ & 718 & 6,4 & 81,8 \\
\hline $24-29$ & 610 & 8,5 & 71,1 \\
\hline $30-39$ & 611 & 11,0 & 69,8 \\
\hline $40-49$ & 301 & 13,9 & 71,2 \\
\hline $50-69$ & 166 & 22,1 & 68,1 \\
\hline
\end{tabular}

Les hommes, dont on a vu la plus grande réserve, ont des relations de confidence très légèrement plus anciennes que celles citées par des femmes.

En comparant l'accroissement de l'âge moyen des enquêtés à l'accroissement de l'ancienneté des relations qu'ils citent, on fait apparaître que les liens "vieillissent" toujours moins que les individus : il n'existe pas un "stock" de relations qui vieillirait avec les individus et s'épuiserait progressivement (ce que la diminution de la taille du réseau avec l'âge laisse spontanément supposer), mais un renouvellement permanent (FERRAND, 1989a). Ce n'est qu'à partir de 50 ans que ce renouvellement faiblit. L'ancienneté des relations croît de façon régulière avec l'âge du citeur, mais pas selon la même pente ${ }^{21}$ : à tous âges on se refait des relations nouvelles, mais plus on avance en âge, moins la proportion de ces relations récentes est importante, conduisant l'ancienneté moyenne des relations à s'accroître.

Pour chaque classe d'âge, le coefficient de variation indique la diversité relative des logiques relationnelles : il oppose les plus jeunes à tous les autres. Ceci relève du contraste le plus important aux âges jeunes entre les relations "depuis toujours" qui ont une durée d'au moins 18 ans et les relations plus récentes. La stabilité de cette dispersion relative montre qu'aux âges avancés les écarts bruts sont plus importants, mais que globalement les variations interindividuelles sont homologues au cours de la vie.

\footnotetext{
${ }^{21}$ Nous considérons que ces logiques relationnelles sont peu affectées par des changements historiques et négligeons ici l'effet "génération".
} 
TABLEAU 10. - Accroissement de la durée des relations amicales

en fonction de l'âge des enquêtés

$\begin{array}{cccc}\text { Age } & \begin{array}{c}\text { Age } \\ \text { moyen }\end{array} & \begin{array}{l}\text { Veillis- } \\ \text { sement des } \\ \text { enquêtés }\end{array} & \begin{array}{l}\text { Accroissement } \\ \text { de la durée } \\ \text { des relations }\end{array} \\ 18-23 & 20,7 & +5,7 & +2,1 \\ 24-29 & 26,4 & +7,6 & +2,5 \\ 30-39 & 34, \odot & +9,6 & +2,9 \\ 40-49 & 43,6 & +14,4 & +8,2 \\ 50-69 & 58, \odot & \end{array}$

Le passage de la trentaine à la quarantaine fait apparaître un contraste selon le sexe des citeurs : les relations des hommes ont vieilli de 4,4 ans contre 1,6 ans pour celles des femmes. Ces dernières renouvellent donc leurs partenaires de discussion de façon beaucoup plus marquée : diminution des tâches liées aux enfants, reprise d'un travail, peut-être essoufflement de la vie couple, ces diverses ruptures peuvent les conduire à inaugurer de nouvelles relations de confidence.

Les relations de confidence décrites sont citées par des individus qui ont des réseaux variés : certains ont une seule relation, d'autres plusieurs. Au sein de la logique générale qui veut qu'à tous les âges on se recrée de nouvelles relations, un réseau plus étoffé peut correspondre à un "conservatisme" relationnel : on garderait plus longuement des liens qui, ainsi s'accumuleraient. En fait le jeu est inverse. Les relations appartenant à des réseaux de trois personnes sont en moyenne de deux ans plus récentes que les relations uniques : avoir plus de confidents, c'est aussi les renouveler plus rapidement. L'expansivité, traduite par une plus grande taille du réseau, s'accompagne donc d'un régime plus dynamique où création et abandon de relations s'accélèrent.

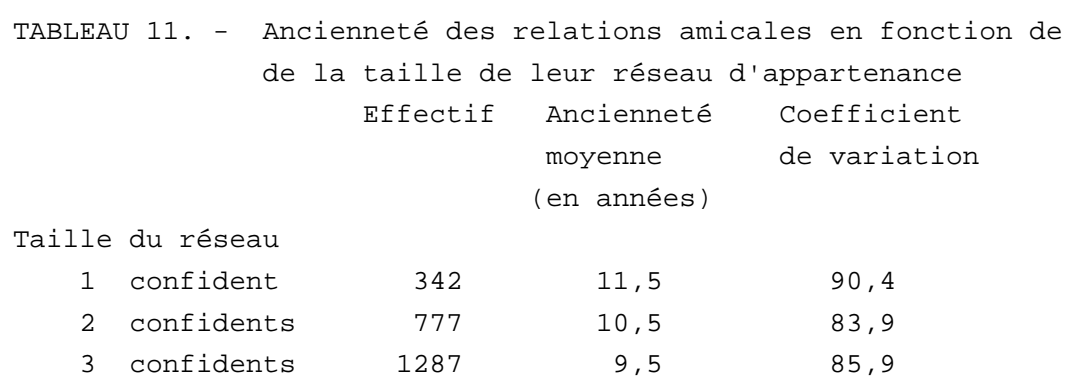

Ce lien entre expansivité et renouvellement n'est pas homogène sur l'ensemble du cours de la vie : jusqu'à trente ans la taille des réseaux n'introduit pas de différences sensibles dans l'ancienneté des liens amicaux. Après cet âge, les écarts d'ancienneté entre liens uniques et liens appartenant à des réseaux de trois confidents s'accroissent pour passer de 2 à 6 ans. C'est à partir de la trentaine qu'apparaissent des logiques contrastées: d'un côté des réseaux "monoconfidents" constitués par un lien amical durable, de l'autre, des réseaux de "multi-confidents" qui se renouvellent plus souvent.

Si les réseaux amicaux des hommes sont en moyenne plus petits que ceux des femmes (1,4 contre 1,6 ), ils manifestent le même rapport entre expansivité et dynamique : un plus grand nombre d'amis s'accompagne pour eux aussi d'un caractère plus récent des liens. 


\subsubsection{UNE LOGIQUE INTERNE DES PROCESSUS RELATIONNELS : ANCIENS ET NOUVEAUX CONFIDENTS}

Ayant vu comment l'âge et le sexe des citeurs (propriétés des individus) et le nombre de confidents cités (propriété formelle du réseau) sont en rapport avec l'ancienneté des liens, nous voudrions examiner si des propriétés de la relation elle-même sont liées de manière indépendante à son ancienneté22. Ce type d'analyse repose sur une hypothèse qui donne le plus d'importance à une logiques intrinsèque des relations qu'aux propriétés des individus qui les ont citées. Ce sont des propriétés formelles de la relation qui en expliquent le devenir.

L'âge de l'individu et de son confident forment des limites objectives aux durées maxima, et influent globalement sur les durées moyennes réelles. Pour analyser la stabilité des relations en neutralisant l'âge des enquêtés, nous avons construit une variable de durée relative qui tient compte de la distribution de l'ancienneté des liens par classe d'âge : les relations du premier quartile seront dites "récentes", celles du quatrième seront dites "anciennes", les autres "intermédiaires"23.

A tous âges, les relations amicales (relativement) les plus récentes sont citées préférentiellement par des femmes. Ce sont des amis inclus dans des réseaux de trois confidents, et on les rencontre chaque semaine. Les amis de même sexe et de même âge sont, au contraire, sous-représentés. Les amitiés les plus anciennes sont formées par des hommes ou des femmes, mais avec des confidents de même sexe et de même âge (plus ou moins 3 ans). La fréquence des contacts est indifférente. Et l'homophilie professionnelle est rare : au cours de la vie, des trajectoires professionnelles peuvent diverger sans remettre en cause ces liens fondamentaux. Enfin, le fait qu'on ait eu "des relations amoureuses ou sexuelles" avec le confident cité ne pèse pas significativement sur l'ancienneté des connaissances.

Ainsi les propriétés formelles d'homophilie et la fréquence des contacts sont liées à la chance de faire durer des liens amicaux. Ces différences dans les caractéristiques du lien montrent que, globalement, tous les liens récents ne sont pas destinés, avec le temps, à devenir anciens : seules résisteront les relations amicales caractérisées par la plus grande proximité identitaire des sujets selon les dimensions culturelles de sexe et d'âge ${ }^{24}$.

L'analyse de la totalité du réseau des relations amicales des individus, et de l'histoire de ces relations, réalisée par C.BIDART ${ }^{25}$ permet de préciser ces deux modèles.

D'un côté, "des amitiés nées dans l'enfance ou la jeunesse" auront des chances d'être durables si "les partenaires bénéficient d'une certaine concordance dans le déroulement de leurs cycles de vie respectifs : ils se sont mariés en même temps, ont fait des enfants en même temps... On voit également des amis, un temps séparés, se retrouver lorsque le retardataire rattrape son

\footnotetext{
22 La technique utilisée est la régression logistique (GLAUDE, 1983, VERGER, 1983) qui permet de mesurer des effets "toutes choses étant égales par ailleurs". Elle s'applique aux relations amicales, étant exclues les relations émises par des personnes n'ayant pas déclarées leur professions, les relations confidentes dont la profession est absente et celles qui ne mentionnent pas la fréquence des interactions ( $\mathrm{N}=2336$ relations sur 2406 relations amicales citées)..

23 Nous remercions Marie-Odile LEBEAUX (LASMAS) pour ses conseils méthodologiques.

24 On trouvera dans FERRAND, 1985 un modèle théorique d'interprétation de ce type d'amitié.

25 Interviews approfondies, auprès de personnes de milieu "employé", reconstituant l'origine des amitiés et le rôle particulier des échanges de confidences. Nous citons une note intermédiaire de travail de Novembre 1992.
} 
ami... Les relations qui survivent sont d'autre part celles qui ont été, à un moment donné de leur cursus, davantage personnalisées, distinguées de l'ensemble du groupe initial par un rapport particulier et particularisant. Ce "moment fondateur" d'une interpersonnalité privilégiée se situe le plus souvent à la suite d'un événement à caractère dramatique."

De l'autre "des amitiés d'adulte sont plus rapidement différenciées, détachées de leur contexte initial de rencontre... C'est le plus souvent une situation exceptionnelle ou un événement dramatique, qui a favorisé, voire provoqué, cette élection... Là peuvent se révéler des points communs, des proximités en termes de personnalité qui, davantage que pour les amis d'enfance, "font le lien"... Ces relations sont moins homogènes du point de vue de l'âge et du sexe, mais le sont davantage du point de vue du statut socio-professionnel, plus stabilisé et défini à ces âges, et plus structurant... Ce sont aussi les relations qui "accompagnent" le plus la vie quotidienne et qui bénéficient d'une fréquentation plus importante que les amis d'enfance, souvent éloignés géographiquement. Les confidences sont là, plus qu'une occasion annuelle de "faire le point", une façon de suivre l'intimité personnelle au jour le jour."

TABLEAU 12. - Durée relative des relations amicales en fonction

de quelques propriétés des relations et du réseau

\begin{tabular}{|c|c|c|c|c|}
\hline \multirow[b]{2}{*}{ Variables retenues } & \multicolumn{2}{|c|}{ Durées récentes } & \multicolumn{2}{|c|}{ Durées anciennes } \\
\hline & $\begin{array}{l}\text { Paramètre } \\
\text { estimé }\end{array}$ & Probabilité( & $\begin{array}{l}\text { Paramètre } \\
\text { estimé }\end{array}$ & Probabilité) \\
\hline \multicolumn{5}{|l|}{ Sexe ego } \\
\hline femme & $\odot, 2491$ & 0,0116 & $-\odot, 1615$ & \\
\hline Homme ( ) & & & & \\
\hline \multicolumn{5}{|l|}{ Nombre confidents } \\
\hline 1 & $-0,2913$ & & $\odot, 6110$ & \\
\hline \multicolumn{5}{|l|}{2} \\
\hline 3 & $\odot, 2683$ & $\odot, 0152$ & $\odot, 0124$ & \\
\hline \multicolumn{5}{|l|}{$\begin{array}{l}\text { Relations sexuelles } \\
\text { ou amoureuse avec le } \\
\text { confident }\end{array}$} \\
\hline oui & $-0,0117$ & & $-\odot, 0913$ & \\
\hline \multicolumn{5}{|l|}{ non } \\
\hline non réponse & $-\odot, 3970$ & & $-\odot, 3777$ & \\
\hline \multicolumn{5}{|c|}{ Fréquence des rencontres } \\
\hline moins une semaine & $\odot, 5406$ & $\odot, 0005$ & $-\odot, 1734$ & \\
\hline moins un mois & $\bullet, 3186$ & & $-\odot, 0344$ & \\
\hline plus d'un mois & & & $-\odot, 4164$ & \\
\hline \multicolumn{5}{|l|}{$\begin{array}{l}\text { Relations homophiles } \\
\text { selon : }\end{array}$} \\
\hline sexe & $-\odot, 5962$ & $\odot, 0 \odot \odot 1$ & $\odot, 5464$ & $\odot, 0 \odot \odot 1$ \\
\hline âge & $-0,5293$ & $\odot, 0001$ & $\odot, 7041$ & $\odot, 0001$ \\
\hline CSP & 0,0238 & & $-\odot, 4164$ & 0,0001 \\
\hline
\end{tabular}

Les modalités des variables en italique constituent la situation de référence. $\mathrm{Ne}$ sont mentionnées que les probabilités <0.01. 


\subsection{Conclusion}

L'analyse des "réseaux personnels" est paradoxale : fondée sur un paradigme qui privilégie l'interaction entre deux partenaires, et si possible les effets structuraux dans un système complet de relations, elle procède cependant par enquête auprès d'un seul terme de la relation. Ce type d'enquête "en base individu" est, évidemment, plus aisément praticable que l'observation de toutes les relations dans un ensemble donné d'individus, mais l'analyse doit s'intéresser essentiellement aux propriétés formelles des liens pour surmonter le paradoxe : on fait alors ressortir l'existence de processus répondant à des modèles relationnels spécifiques, résultat plus conforme au paradigme interactionniste.

Dans le même esprit, la description des "choix" peut nourrir une sociographie des "souscultures relationnelles" ou des "modes de sociabilité" propres à différents milieux, pensés comme des univers séparés. Elle peut aussi, comme nous avons tenté de l'esquisser, ouvrir sur une vision plus structurale: les choix relationnels sont les opérateurs de connexion entre différentes régions du champ social. D'une part tous les choix sont formellement interdépendants, rétroagissants : il s'agit moins d'une approche différentielle des normes de sociabilité, que d'une vision structurelle des "barrières et niveaux"26. D'autre part cette vision synthétise les liens, donc les canaux d'échanges, entre catégories d'acteurs : échanges de paroles ici, permettant de mieux comprendre des logiques d'information, échanges amoureux ailleurs, potentiellement porteurs d'infection virale, dans le contexte du sida.

\section{REFERENCES BIBLIOGRAPHIQUES}

ALLAN (G.H.), (1979), A sociology of friendship and kinship. London, George Allen and University of Wisconsin.

BIDART((Claire), (1991), "L'amitié, les amis, leur histoire". Sociétés Contemporaines, n5, 21-

42)

BOZON (Michel), HERAN (François), (1987), "L'aire de recrutement du conjoint". Données sociales, 338-347.

BURT (Ronald S.), (1985), "Network items and the General Social Survey". Social Network, $\mathrm{n}^{\circ}$ 6, 293-339.

DEGENNE (Alain), 1978, "La construction et l'analyse des réseaux sociaux". L'Année Sociologique, $\mathrm{n}^{\circ} 29,1978$

FERRAND (Alexis), (1985), Amis et Associés. Paris, CESOL, Fascicules 1,2,6 .

FERRAND (Alexis) (ed.), (1987), Un niveau intermédiaire, les réseaux sociaux. Actes du séminaire organisé à l'IRESCO par le CESOL, PARIS, CESOL-A.FERRAND 103 p.

FERRAND (Alexis), (1989a), "Connaissances passagères et vieux amis. Les durées de vie des relations interpersonnelles". Revue Suisse de Sociologie, n²2, 431-439.

FERRAND (Alexis), (1989b), "A wholistic approach of interpersonal relations" . Communication INSNA Sunbelt Conference, TAMPA (FL) 17 p.

FERRAND (Alexis), MOUNIER (Lise), (1990), Relations sexuelles et relations de confidence : Analyse de réseaux. Recherche réalisée pour l'ANRS, LASMAS-CNRS, 175 p.

\footnotetext{
${ }^{26}$ Les tables de mobilité ont une logique un peu analogue.
} 
FISCHER (C.S.), (1982), To dwell among friends ; personal networks in town and city. The University of Chicago Press, $451 \mathrm{p}$.

GLAUDE (M.), (1983), "Le coût de l'enfant". Economie et statistique., nº 155.

HERAN F. La sociabilité, une pratique culturelle. Economie et statistique, 1988, n²16, p.3-21.

LAZARSFELD (Paul), MERTON (Robert K.), (1954), "Friendship as a social process". In BERGER (M.), VAN (A.), Freedom and control in modern society. Nostrand Princeton.

LAZEGA (Emmanuel), (1992), "Les relations de travail des Français vues par l'enquête "Contacts entre les personnes". Rapport au PIRTTEM. 50 p.

MARSDEN (P.V.), (1987), "Core discussion, network of Americans". American Sociological Review, vol. 52, 22-131.

MATTHEWS (S.), (1986), Friendship through the life course : oral biographies in old age. Beverly Hill, Sage.

PETO (D.), REMY (J.), VAN CAMPENHOUDT (L.), HUBERT (M.), (1992), Modes d'adaptation au risque du Sida dans les relations hétérosexuelles. Bruxelles, Centre d'Etudes Sociologiques.

REICHMANN (S.), (1991), "Impact du chômage sur la santé mentale". Sociétés contemporaines, n5, 99-116.

ROGERS (E.M.), BHOWMIK (D.K.), (1971), "Homophily - heterophily : relational concepts for communication researches". Public Opinion Quaterly, n³4, 523-538.

ROSE (Arnold M.), (1962), "A systematic summary of symbolic interaction theory". in A. M. ROSE (ed.), Human behavior and social processes: an interactionist approach. London : Houghton Mifflin.

VERGER (D.), (1983), "L'achat d'un logement ne va pas sans achat d'équipement. Annexe méthodologique : le modèle "logit". Economie et statistique , n 161. 